\title{
Generation of high-quality signals for optical sensing using DFB lasers injection locking
}

\author{
L.THEVENAZ (luc.thevenaz@epfl.ch), D.ALASIA, S.LE FLOCH, J.TROGER \\ Laboratory of Nanophotonics and Metrology (NAM), \\ Swiss Federal Institute of Technology, CH-1015 Lausanne, Switzerland
}

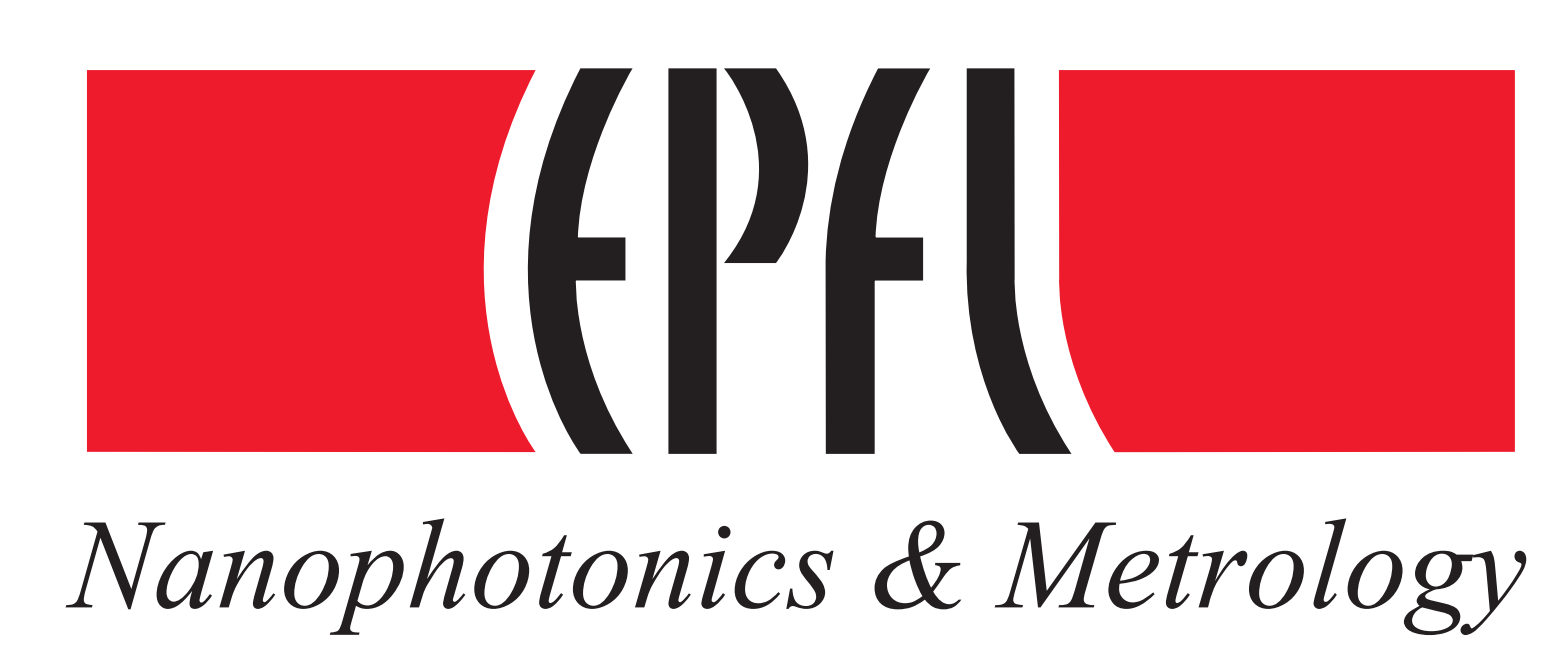

Injection locking of two DFB semiconductor lasers opens new possibilities to generate effective signals for optical sensing, in order to reach better performances. The principle is in essence very simple: a small fraction of the light of an isolated master laser is injected into the cavity of a slave laser. If the frequency difference between the 2 lasers lies

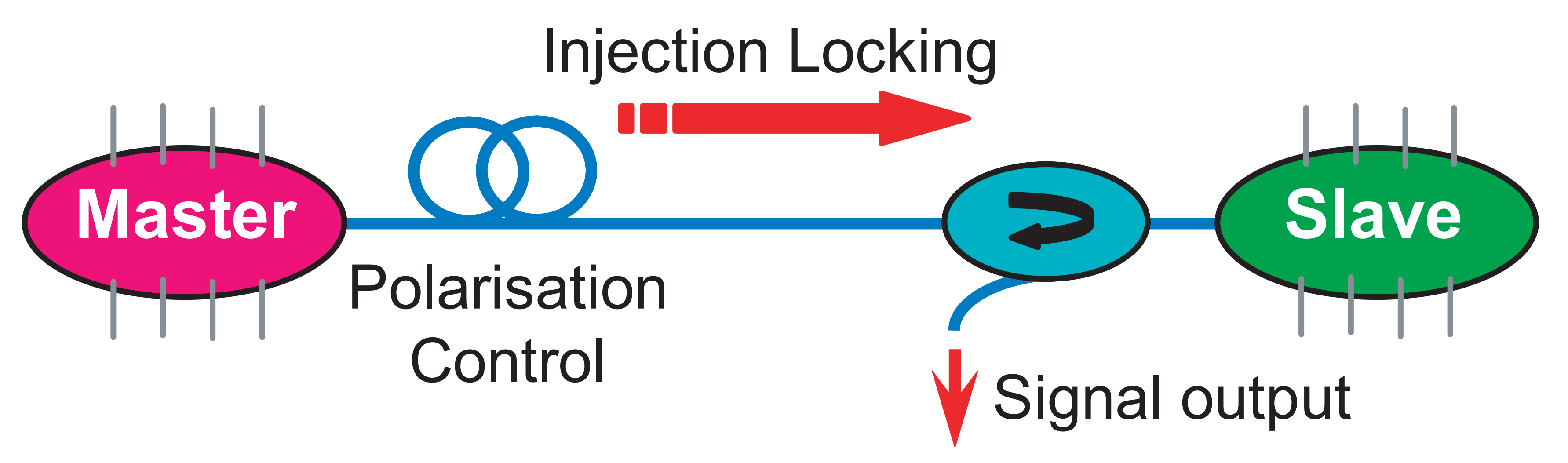

Pure AM modulation

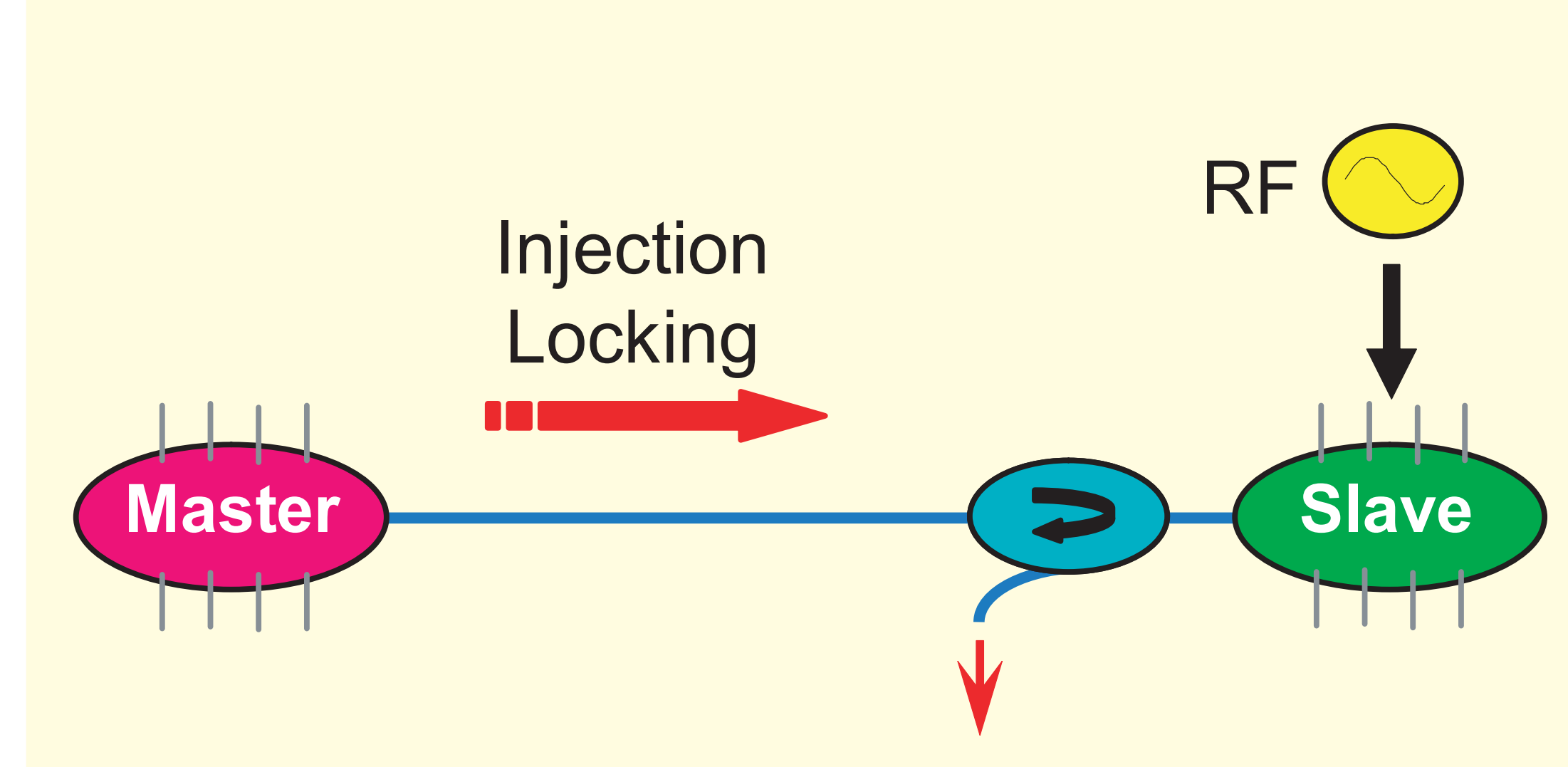

The master laser is operated in $\mathrm{CW}$ mode and the current of the slave is modulated at the modulation frequency. The carrier of the slave locks on the master emission line, resulting in no frequency dithering and therefore no unwanted FM modulation.
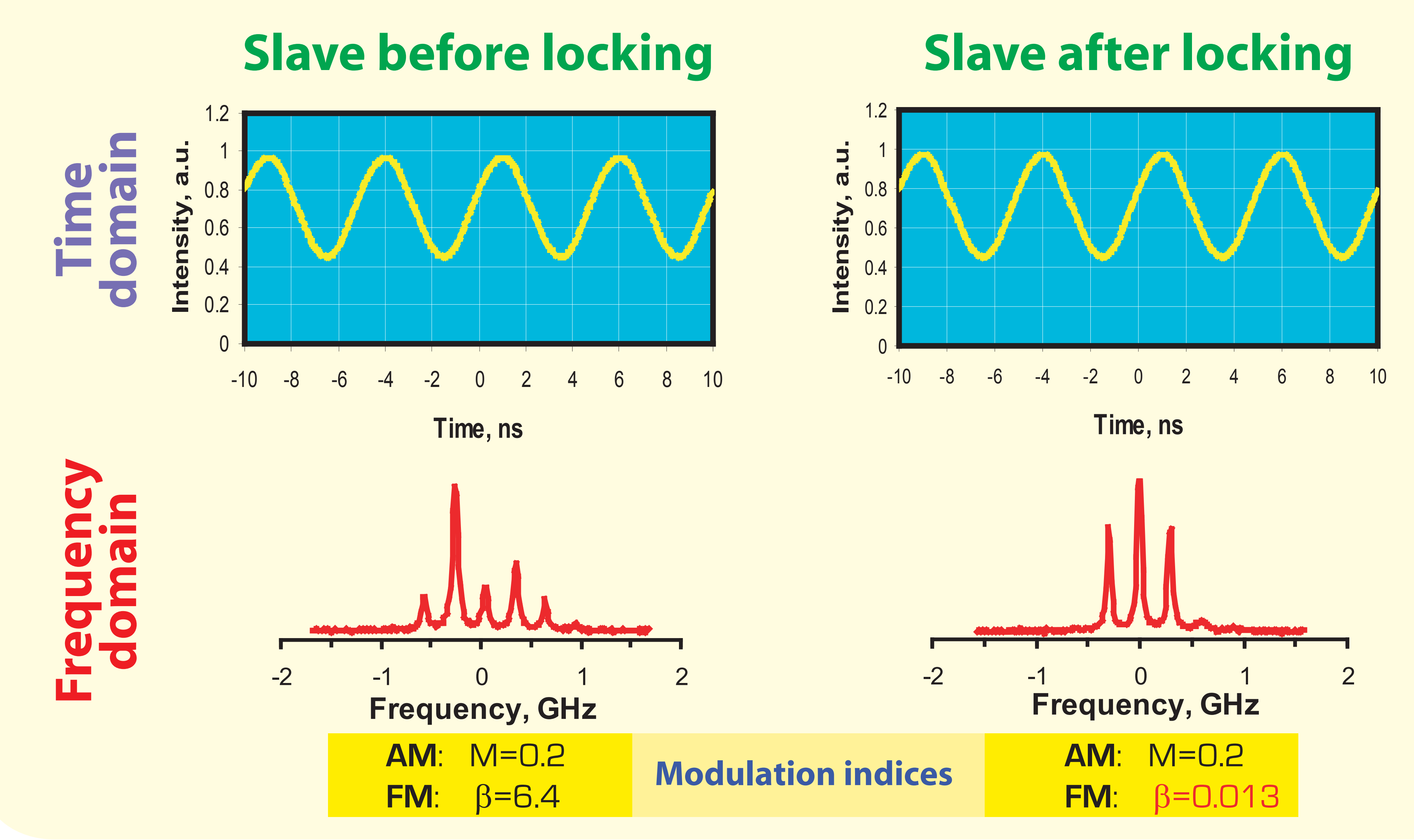

\section{Optical frequency shifting}

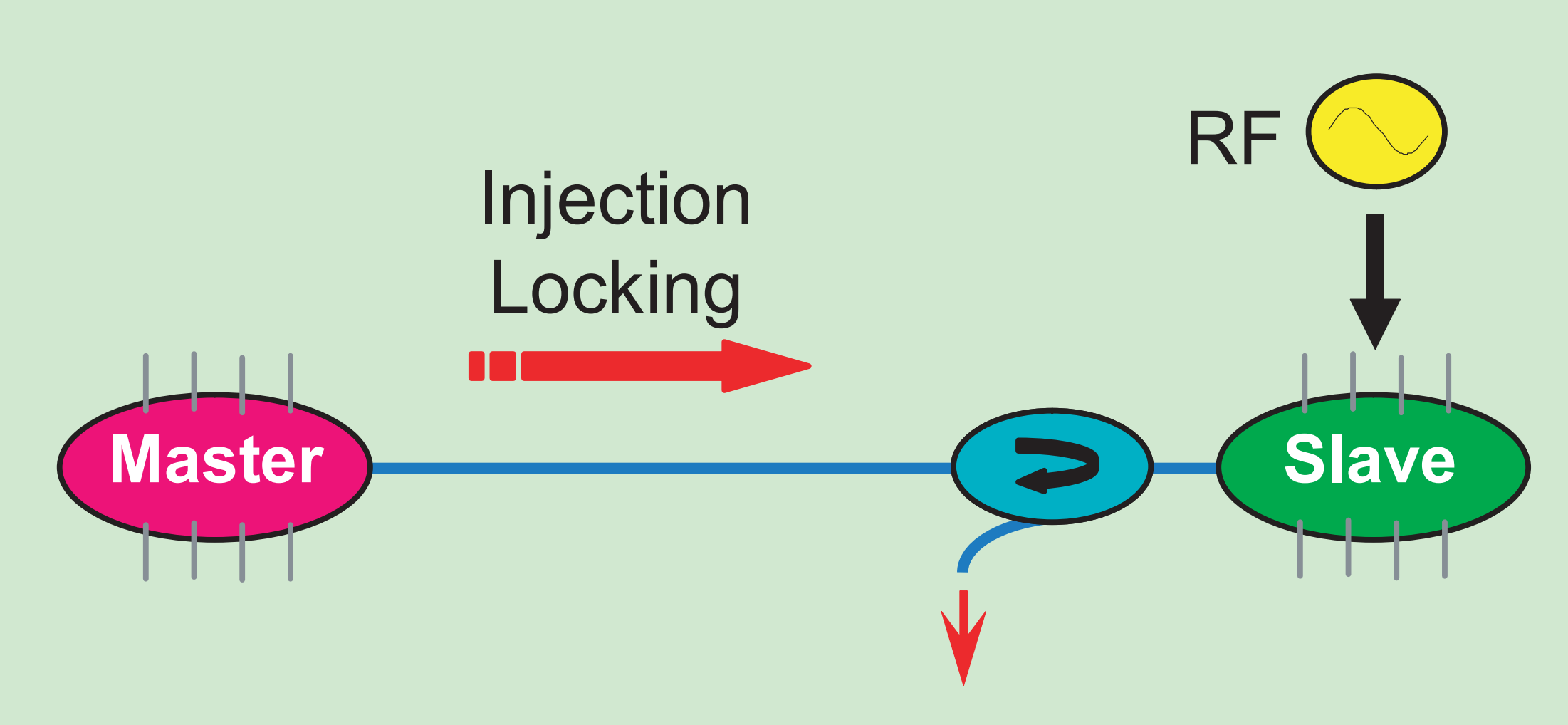

This is simply achieved by modulating the current of the master laser and by locking the slave on one of the modulation sidebands.

The frequency difference of the 2 lasers is thus perfectly stable and a pure beat note is obtained. within the locking range (typ. $1 \mathrm{GHz}$ ), the slave laser leaves its own frequency to be faithfully phase-locked on the master laser frequency.

Smart configurations make possible the generation of a wide range of signals that are difficult to obtain using standard configurations and often with qualities exceeding those obtained using external modulators.

\section{Pure FM modulation}

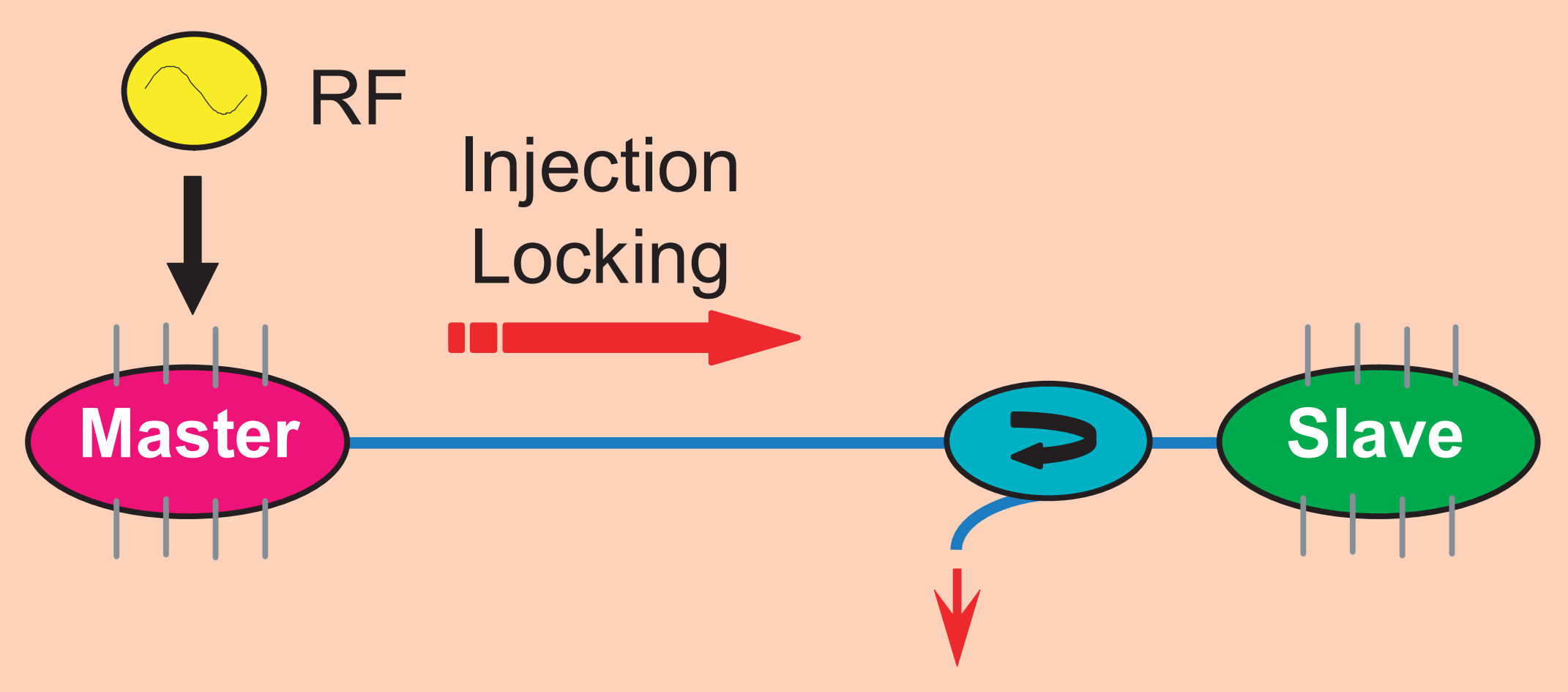

The current of the master is modulated while the slave is operated in CW mode. The instantaneous frequency of the slave locks onto the instantaneous emission line of the master. The slave shows no significant change of its emission power resulting in a pure frequency dithering.
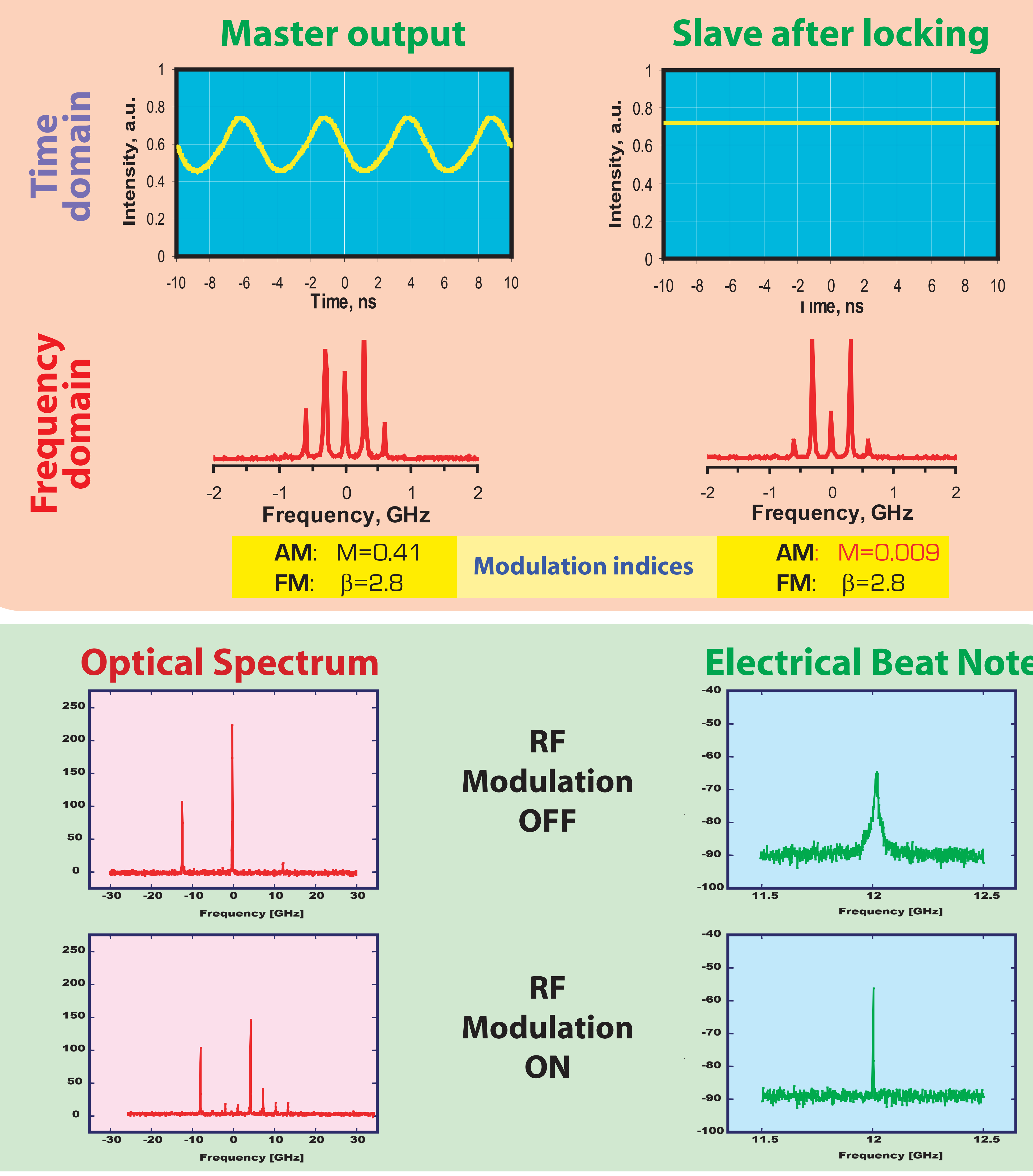

\section{Optical frequency sweeping}

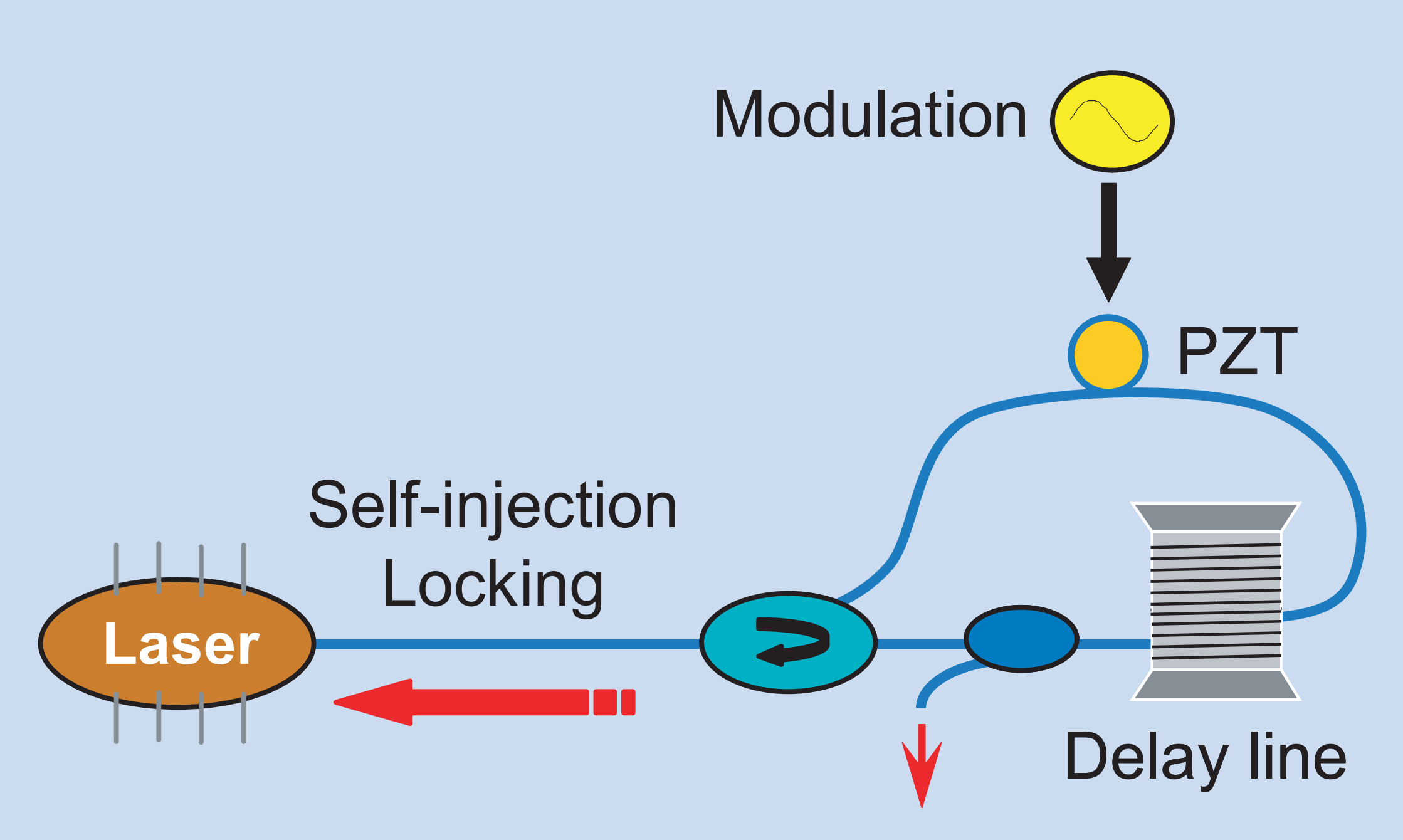

The slave laser is locked on its own emission, after phase modulation and a time delay resonant with the modulation. The frequency variations then add up constructively at each round trip until the sweep amplitude corresponds to the locking range.

The optical spectrum on the right shows the typical aspect of a broadband FM modulation.

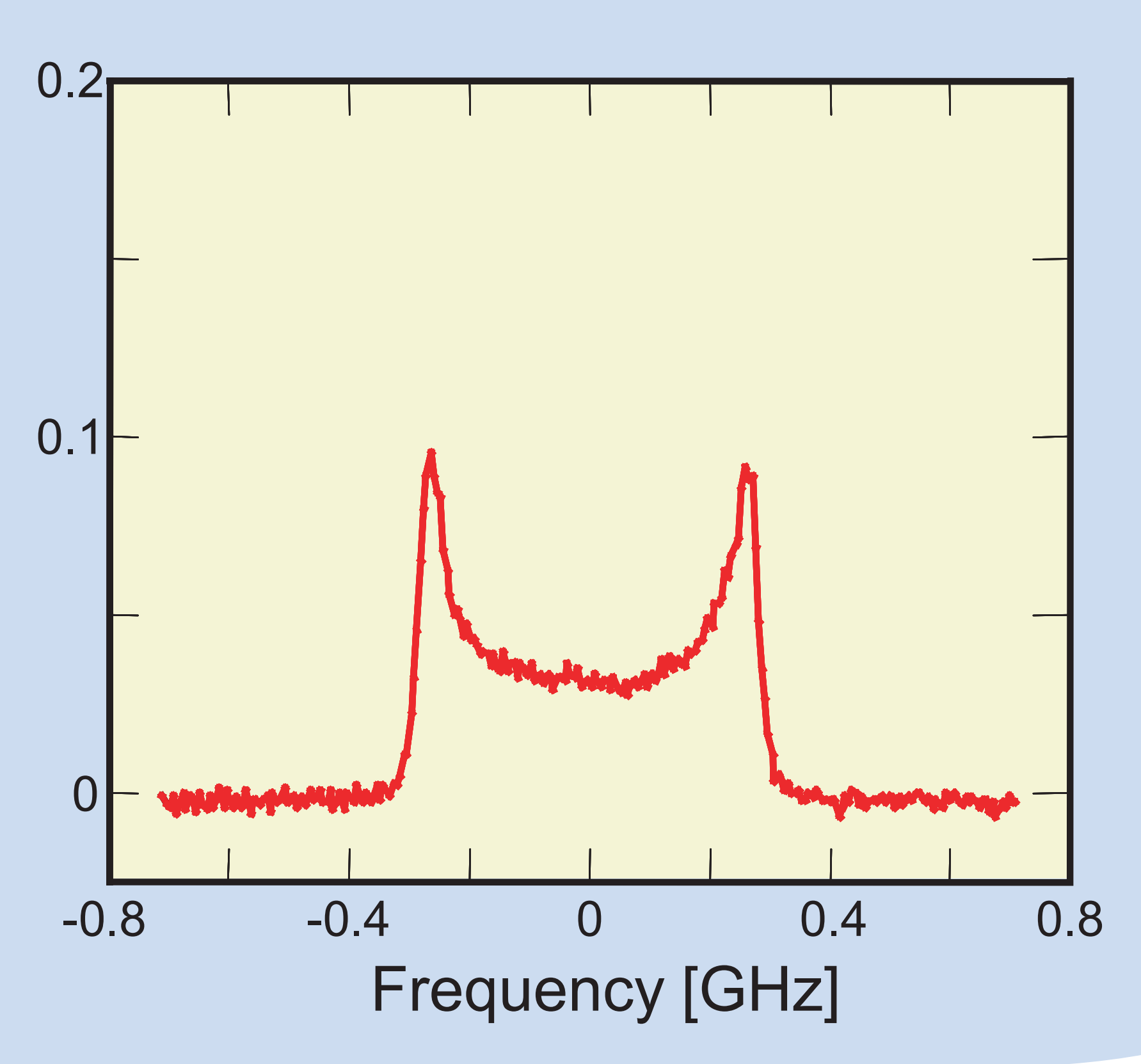

